\title{
Consumption of Salt Rich Products: Impact of the UK Reduced Salt \\ Campaign
}

\author{
Abhijit Sharma, Salvatore di Falco ${ }^{\dagger}$ and Iain Fraser $^{\ddagger}$
}

October 2018

\begin{abstract}
This paper uses a leading UK supermarket's loyalty card database to assess the effectiveness and impact of the 2004 UK reduced salt campaign. We present an econometric analysis of purchase data to assess the effectiveness of the Food Standard Agency's (FSA) 'reduced salt campaign'. We adopt a general approach to determining structural breaks in the time series of purchase data, using unit root tests whereby structural breaks are endogenously determined from the data. We find only limited evidence supporting the effectiveness of the FSA's reduced salt campaign. Our results support existing findings in the literature that have used alternative methodologies to examine the impact of information campaigns on consumer choice of products with high salt content.
\end{abstract}

JEL codes: Q12, Q31, I12

Keywords: structural breaks, salt consumption, low salt campaign effects

${ }^{*}$ Corresponding author: Abhijit Sharma, Bradford University School of Management, Bradford BD9 4JL, UK. Ph: +44-1274-234781; Email: A. Sharma12@brad.ac.uk. Acknowledgement: Research funded by the UK Food Standards Agency (FSA Project Code: D03008). We would also like to acknowledge help received from Andrew Fearne in obtaining the dataset used in this paper.

${ }^{t}$ University of Geneva, Switzerland. Email: salvatore.difalco@unige.ch

${ }^{\dagger}$ University of Kent, Canterbury, UK. Email: I.M.Fraser@kent.ac.uk. 


\title{
Consumption of Salt Rich Products: Impact of the UK Reduced Salt Campaign
}

\author{
15 Oct 2018
}

\begin{abstract}
This paper uses a leading UK supermarket's loyalty card database to assess the effectiveness and impact of the 2004 UK reduced salt campaign. We present an econometric analysis of purchase data to assess the effectiveness of the Food Standard Agency's (FSA) 'reduced salt campaign'. We adopt a general approach to determining structural breaks in the time series of purchase data, using unit root tests whereby structural breaks are endogenously determined from the data. We find only limited evidence supporting the effectiveness of the FSA's reduced salt campaign. Our results support existing findings in the literature that have used alternative methodologies to examine the impact of information campaigns on consumer choice of products with high salt content.
\end{abstract}

JEL codes: Q12, Q31, I12

Keywords: structural breaks, salt consumption, low salt campaign effects 


\section{Introduction}

Conditions such as high blood pressure are considered among the most important risk factors for cardiovascular disease. For instance the World Health Organization (2014) proposed a global target of a reduction in the incidence of high blood pressure by $25 \%$. In addition, it is also recognised by the WHO (2014) that high levels of salt intake also contribute to increased levels of blood pressure as well as increased risks of coronary heart disease and strokes. For this reason they also propose a global target of a 30\% reduction in salt consumption. These targets are central to the reduction of non-communicable diseases (NCDs) globally. Within the UK there is a long standing policy effort to achieve related reductions in salt. There have been a number of public interventions aimed at raising awareness of salt as a public health issue, and inform consumers how to lower their salt intakes. For example, the UK Department of Health and the Food Standards Agency (FSA) implemented a combined policy to prompt the food industry to add less salt in food and to persuade the public to consume less salt (He and MacGregor, 2009).

In this paper, we focus on information aimed at encouraging consumers to consume food that contains lower amounts of salt. Specifically, we examine econometrically the 2004 FSA 'low salt campaign'. This campaign attempted to raise public awareness of the implications of high salt intake on their health and wellbeing. The campaign was implemented over a period of time and we focus on the first three stages of the campaign. The first phase was launched in September 2004, with the aim of ensuring that consumers were aware of why too much salt is bad for their health. In the second phase of the campaign (launched in October 2005) consumers were encouraged to check food labels for information on the salt content and to raise awareness of the aim to eat no more than $6 \mathrm{~g}$ of salt a day. The third phase was launched in March 2007. This part of the campaign focused on the message that $75 \%$ of the salt consumers' eat is already in everyday foods.

Our research employs the Tesco Clubcard supermarket loyalty card database. Eighty percent of Tesco transactions go through Clubcard and the database covers 1.2 million UK consumers. The data we employ spans two years. We use data for weekly volumes of sales from 26 September 2005 to 17 September 2007. We make use of information on volumes and sales of high salt products and their low salt counterparts, focusing in particular on typical items contained within the consumption bundles chosen by the average consumer such as crisps, bacon and soups.

Our main research question and the associated hypothesis relates to the effectiveness or otherwise of the low salt campaign. We expect the low salt campaign to have had a significant effect on the consumption of high salt content food items such that time series data for these products will exhibit a structural break i.e. show a change in the level of consumption after the advertising campaign has been delivered. The time period that we include for consideration after the advertising campaign has been determined by following the empirical literature within health economics and related areas. Empirical work within consumer behaviour and advertising strongly indicates that a time window of about 10-12 weeks is associated with the significant impact on consumption decisions, because in non-involved or low-involvement purchasing decisions there is likely to be a degree of persistence in behaviour (see for example, Mela et al., 1997). The time frame of data we employ gives us the opportunity to assess whether any changes in purchases of high salt content food are related to the phases of the information campaign. In particular, the relatively high frequency (weekly) of data available to us allows for the identification of breaks or changes in demand. Thus, the main objective of our research is to determine whether the 'No More Than $6 \mathrm{~g}$ of Salt a Day' campaign led to a change in consumer behaviour and consumption of some high salt food items.

To undertake this research, we employ time series econometric methods to test for structural breaks in our data series. It is well known that structural breaks should be allowed for whilst simultaneously testing for unit roots. If pre-determined breaks are imposed, it might lead to incorrect specification of break points. One solution for this is to employ unit root tests whereby structural breaks are endogenously determined from the data. Such unit root tests are more likely to avoid spurious rejections of the unit root null in the presence of structural breaks. To this effect, we employ several 
such tests following Zivot and Andrews (1992), Bai and Perron (1998, 2003), Lee and Strazicich (2003) and Lee et al. (2006). If we find evidence of structural breaks, we then examine whether or not these breaks are associated with the phases of implementation of the health campaigns. The econometric procedures we employ allow for the identification of significant changes in sales of products that are high in salt and they enable us to assess the impact of health related announcements. Thus, our empirical analysis assesses the effectiveness of the FSA 'reduced salt campaign' in terms of changes in purchase behaviour.

Based on our analysis, we find that among all products considered (i.e. crisps, soups, and bacon), only bacon shows breaks potentially related to the FSA campaign. For instance, for smoked bacon and unsmoked bacon, we observe that there is a downward trend generally in the consumption of both smoked and unsmoked bacon. Two breaks are identified. The first one takes place six weeks after the second round of announcements, but this is not statistically significant. The second break is statistically significant, and it takes place in proximity to the third campaign. More precisely, it occurs three weeks after the third round of the FSA campaign. We can therefore tentatively conclude that the campaign appears to have been effective in inducing and maintaining the downward trend in consumption of specific food items which are high in salt. However, there is less evidence that the consumer information campaigns have resulted in a general reduction in the purchase of food items that contain high quantities of salt. These results are in keeping with existing results reported on food information campaigns (e.g. Lui et al., 2014). Therefore, given these results it is reasonable to conclude that an information campaign should only be viewed as one part of an effective policy response to a problem such as that related to salt consumption.

\section{The FSA Low salt campaign and antecedent research}

The UK FSA launched its low salt campaign towards the end of 2004. Its main objective was to achieve lower levels of salt consumption by the UK population. The campaign was comprised of two components. The first one was aimed at raising awareness relating to the recommended maximum daily salt intake by employing a series of advertisements. Subsequently this message was reinforced in further related campaigns that were run in later years. The advertisement campaign itself made use of a cartoon character called 'Sid the Slug' who pointed out the health risks associated with high salt consumption. The FSA also employed 'talking foods' and 'full of it' to highlight the health risks associated with excessive salt intake. This FSA campaign also involved additional steps such as use of peer education and social awareness programmes. The second component involved concerted efforts to co-ordinate reduction of salt levels in processed food by working in collaboration with the food industry (Griffiths et al., 2017).

In terms of existing research with regard to the FSA campaign there are number of relevant studies to consider. These studies can be divided into research that examines the FSA campaign specifically and the examination of salt reduction policies in general. With regard to studies conducted focussing on the FSA campaign there have been efforts made to distinguish between salt added at the table as opposed to the consumption of prepared food with a given level of salt included. For example, Sutherland et al. (2013) use a large individual level repeated cross-section data set drawn from the Health Survey of England (covering 1997 to 2007). This data set records adult use of salt at the table. Sutherland et al. (2013) report a reduction salt use at the table for the period they examine and attribute this in part to salt reduction campaigns.

In related research, Shankar et al. (2013) conclude that the FSA low salt campaign was effective and that it resulted in a decrease of salt consumption by approximately $10 \%$. This research extended Sutherland et al. (2013) by combing the health survey data with data on food prices taken from the Expenditure and Food Survey and constructing a pseudo-panel and estimating panel fixed effects specification.

Most recently, Griffith et al. (2017) conclude that it was product reformulation that drove the observed reduction in salt and not changes in direct consumption by consumers. The policy approach 
to product reformulation did not force companies to reduce salt, even though government advice clearly argued that this would be a good idea. However, in the absence of government enforcement action, Griffith et al. (2017) argue that a voluntary request by government might be considered as a credible threat for changing industry and firm behaviour. However, it also credible to assume that the food industry realised that the reduced salt informational campaign and plus the implementation of the Traffic Lights nutrition labelling by many leading retailers brought about the change in salt consumption. There is evidence that indicates that consumers would make prepared food choices such that they would avoid the colour red for high salt (Balcombe et al., 2010). As a result food companies reformulated their products and turned a potentially negative request (in terms of compliance through external pressure) from government into a positive outcome from a marketing perspective. Thus, the salt campaign information may have indirectly influenced what consumers might buy and how companies responded. This view is supported by the van Camp et al. (2012) who examine aspects of product formulation directly.

Turning to research that considers information campaigns as part of a larger set of public health approaches to dealing with health issues Collins et al. (2014) in a review of many policy options observes that that information provision can be a cost effective policy option. However, they also note that the actual reduction in salt intake will be quite low and that the impact will not be uniform by product or by household type. In contrast, Nghiem et al. (2015) employ a modelling approach to examine salt reduction policies that includes the mix of policy adopted in the UK. Like Collins et al. (2014) they provide evidence that finds that public information campaigns are cost effective although as might be anticipated policy options that are more demanding on a regulatory basis bring about the largest reductions in salt intake. However, as they observe the political support for such policies is significantly less. Therefore, they conclude that there is a range of policy options and the reductions in salt achieved vary significantly but the likelihood of policy adoption needs to be understood in terms of the political reality. These findings are also supported by Webb et al. (2016) who show that the UK approach to including public health information as part of a policy approach can be a cost effective option if employed globally. However, they also caution against a uniform response across all countries because they cannot be a priori sure that salt reduction will occur in all countries as salt "heavy" products might be more "culturally" important in different parts of the world.

Overall the results of earlier research indicate that there has been a reduction in salt consumption in the UK and that information campaigns can be cost effective although the quantity of salt reduction observed is small. Also, this research draws attention to the important distinction between salt consumed at the table as opposed to salt contained in prepared bought food items. Evidence from the UK suggest that it might be both that have contributed to the overall observed reduction. What is missing in this literature, however, is any evidence from actual purchase data of high content salt foods and whether or not the information campaigns changed existing levels of consumption. Evidence of this would be help in further our understanding of the mechanisms behind the reductions in salt witnessed in the UK over the last decade or so.

\section{Data}

The data we employ in this research is taken from the Tesco Clubcard supermarket loyalty card database. This database collects almost 80 percent of all food transactions for more than one million UK consumers. Our data set covers is weekly sales data for the period 26 September 2005 to 17 September 2007. This means that we have 104 weekly observations. We have specifically focussed on food high in salt content i.e. crisps, bacon and soups.

A summary of the products we examine are presented in Table 1.

Table 1: Food products and data codes

\begin{tabular}{ll}
\hline Food product - description & Code \\
\hline Low salt crisps & crisp.lowsalt (ts1)
\end{tabular}




\begin{tabular}{ll} 
Standard crisps & crisp.standard (ts2) \\
Low salt Kettle chips & kettlechips.lowsalt (ts3) \\
Standard Kettle chips & kettlechips.standard (ts4) \\
Low salt Tesco chips & tescochips.lowsalt (ts5) \\
Standard Tesco chips & tescochips.standard (ts6) \\
Unsmoked bacon & bacon.unsmoked (ts7) \\
Standard bacon & bacon.standard (ts8) \\
Unsmoked Butchers Choice bacon & butcherschoice.baconUS (ts9) \\
Smoked Butchers Choice bacon & butcherschoice.baconS (ts10) \\
Unsmoked Cookstown bacon & cookstown.baconUS (ts11) \\
Smoked Cookstown bacon & cookstown.baconS (ts12) \\
Unsmoked Tesco bacon & tesco.baconUS (ts13) \\
Smoked Tesco bacon & tesco.baconS (ts14) \\
\hline Note: The suffix 'S' denotes standard and 'US' denotes unsmoked
\end{tabular}

As can be seen from Table 1, we have several categories of crisps and chips (low salt crisps, standard crisps, low salt Kettle chips, standard Kettle chips, low salt Tesco chips, standard Tesco chips) plus several categories of bacon (unsmoked bacon, standard bacon, unsmoked Butchers Choice bacon, smoked Butchers Choice bacon, unsmoked Cookstown bacon, smoked Cookstown bacon, unsmoked Tesco bacon, smoked Tesco bacon).

Over the period covered by our dataset there are three rounds of health related announcements. The specific details are presented in Table 2.

Table 2: Announcement Periods

\begin{tabular}{ll}
\hline Round I (R1) & $27 / 09 / 2004-5 / 12 / 2004$ \\
Round II (R2) & $10 / 10 / 2005-30 / 11 / 2005$ \\
Round III (R3) & $12 / 03 / 2007-14 / 05 / 2007$ \\
\hline
\end{tabular}

As can be seen in Table 2 the first round of announcements (R1) lies out of our sample period of data. This means that we focus on the impacts of Rounds 2 and 3 (R2 and R3). The relevant periods for our announcements are weeks 3-10 (R2, viz. 10/10/2005 - 30/11/2005) and weeks 77-86 (R3 viz. 12/03/2007 - 14/05/2007).

\section{Methodology}

We are essentially looking for announcement effects that are captured in consumption behaviour as captured through changes in (reductions in) consumption of food items of interest (high salt foods). Given the health status $\Omega$ of a local population $P_{i}$ (in our case is the catchment area from which this Tesco data is obtained), we assume that the health status of the population is dependent on a set of $k$ variables (some exogenous and some endogenous) such that:

$\Omega_{P_{i}}=f\left(\theta_{i}, \ldots, \theta_{k}\right)$

Assume that one of the variables take the following value $\theta_{j}=\Lambda$, such that $\Lambda \in \Gamma$, where $\Gamma$ represents the information set available to an individual consumer. We assume that governmental health campaigns alter $\Gamma$ and therefore, $\Lambda$, which represents the individual's health related information set. Assuming that the individual is a utility maximising, rational consumer, adverse health related 
information should mean that consumption of food items which reduce life expectancy or increase the probability of contracting illnesses should decline (or even fall to zero for extremely harmful items). Our paper thus aims to identify breaks in consumption data for fourteen salt rich commodities (listed in Table 1) as well as changes in consumption patterns which are associated with the three rounds of FSA's health related announcements under its 'low salt campaign'.

The basic model we use to undertake our analysis can be represented as follows:

$y_{i}=x_{i}^{T} \beta+u_{i}$

where at time $i, y_{i}$ is the observation of the dependent variable, $x_{i}$ is a $k \times 1$ vector of regressors, with the first component usually equal to unity (an intercept), and $\beta_{i}$ is the $k \times 1$ vector of regression coefficients, which may vary over time. The hypothesis being tested is that the regression coefficients remain constant:

$H_{0}: \beta_{i}=\beta_{0}(i=1, \ldots, n)$

against the alternative that at least one coefficient varies over time. In many applications it is reasonable to assume that there are $m$ breakpoints, where the coefficients shift from one stable regression relationship to a different one. Thus, there are $m+1$ segments (where $\mathrm{j}$ denotes the segment index). In practice the breakpoints $i_{j}$ are rarely given exogenously, but have to be estimated. There are a number of approaches that can be used to detect breakpoints. We discuss three of them based on (i) Zivot and Andrews (1992) (ii) Lee and Strazicich (2003, 2004 and (iii) Bai and Perron (1998, 2003).

Zivot and Andrews (1992) propose a unit root testing method which involves an extension of the widely used Dickey and Fuller test, enabling simultaneous estimation of possible breakpoints for the intercept and slope of the trend model. By doing so, Zivot and Andrews (1992) are able to address issues that emerge when structural breakpoints are chosen by simple visual examination of plots of the time series data. Such problems emerge because plots of unit root processes with drifts can often be very similar to processes that are stationary about a trend with a break. The test employed by Zivot and Andrews (1992) is based on recursive estimation of a test regression, where the test statistic is defined as the minimum t-statistic of the coefficient of the lagged endogenous variable. For such a Zivot and Andrews test the null hypothesis is that the time series is integrated (i.e. it has a unit root) and no exogenous structural break exists. The unit-root null hypothesis is rejected if the test-statistic is more negative than the critical value. If the test statistic is more negative than the critical value, then the time series are considered to be trend stationary about a deterministic trend with a single breakpoint.

Lee and Strazicich $(2003,2004)$ propose a unit root test which allows for breaks in the data under both the null and alternative hypothesis in a consistent manner. This procedure is based on a data generating process which can be represented by:

$y_{t}=\delta^{\prime} X_{t}+\epsilon_{t}, \epsilon_{t}=\beta \epsilon_{t-1}+\varepsilon_{t}$

where $X_{t}$ represents a vector of exogenous variables while $\varepsilon_{t}$ is a Gaussian iid error term.

Lee and Strazicich (2003) allow for two changes in level and trend whereby:

$X_{t}=\left[1, t, D_{1 t}, D T_{1 t}, D_{2 t}, D T_{2 t}\right]^{\prime}$

such that $D_{m t}$ and $D T_{m t}$, for $m=1,2$ are dummies with $D_{m t}=1$ for $t \geq T_{B m}+1$ and 0 otherwise. $D_{m t}=t-T_{B m}$ for $t \geq T_{B m}+1$ and 0 otherwise. In this representation, $T_{B m}$ denotes the $m^{t h}$ break date. The data generating process (DGP) permits breaks both under the null $(\beta=1)$ and the alternative hypothesis $(\beta<1)$. In order to estimate the LM unit root test statistic, Lee and Strazicich 
(2003) use the following statistic:

$\Delta y_{t}=\delta^{\prime} \Delta X_{t}+\phi \tilde{Z}_{t-1}+\sum_{i=1}^{k}+\gamma_{i} \Delta \tilde{Z}_{t-m}+u_{t}$

where $\tilde{Z}_{t}=y_{t}-\widetilde{\Phi}_{n}-X_{t} \tilde{\delta}$ for $t=2, \ldots, T$ which is the de-trended series. In this equation, $\tilde{\delta}$ represents the coefficients from the regression of $\Delta y_{t}$ on $\Delta X_{t}, \widetilde{\Phi}_{n}=y_{1}-X_{1} \tilde{\delta}$ and $y_{1}$ and $X_{1}$ represent the first observations. With this method, the lagged terms of $\Delta \tilde{Z}_{t-m}$ are included in order to correct for autocorrelation. From equation [6], we obtain LM test statistics which are given by the $t$-statistics testing for the null hypothesis $\phi=0$. Break dates are then identified by a grid search over all possible dates (once $10 \%$ of endpoints are removed), so that the test statistic is minimised. Critical values can be obtained form Lee and Strazicich (2003).

Bai and Perron's $(1998,2003)$ approach allows multiple $(m)$ break points, whereby there are $m+$ 1 segments in which the regression coefficients are constant, and the model can be rewritten as

$y_{i}=x_{i}^{T} \beta+u_{i} \quad\left(i=i_{\mathrm{j}-1}+1, \ldots, i_{\mathrm{j}}, j=1, \ldots, m+1\right)$

where $j$ denotes the segment index.

The $R$ package "breakpoints" estimates these breakpoints by minimizing the residual sum of squares (RSS) of the equation above. We also have $\mathcal{J}_{m, n}=\left\{i_{1}, \ldots, i_{m}\right\}$ which denotes the set of breakpoints (or the $m$-partition). ${ }^{1} F$ statistics test against a single-shift alternative of unknown timing, i.e., model [7] with $m=1$. Tests against this alternative are usually based on a sequence of $F$ statistics for a change at time $i$ : the OLS residuals $\widehat{u}(i)$ from a segmented regression, i.e., one regression for each subsample, with breakpoint $i$, are compared to the residuals $\widehat{u}$ from the unsegmented model via:

$F_{i}=\frac{\widehat{u}^{T}-\widehat{u}(i)^{T} \widehat{u}(i)}{\widehat{u}(i)^{T} \widehat{u}(i) /(n-2 k)}$

These $F$ statistics are then computed for $i=n_{h}, \ldots, n-n_{h}\left(n_{h} \geq k\right)$ and $H_{0}$ is rejected if their supremum or average or exponential functional is too large.

Hansen (1997) gives an algorithm for computing approximate asymptotic pvalues of these tests, which is implemented in R. A trimming parameter $(\varepsilon)$ can also be chosen, which is defined as minimal segment size either given as a fraction relative to the sample size or as an integer giving the minimal number of observations in each segment. The asymptotic distribution depends on this trimming parameter via the imposition of the minimal length $h$ of a segment, namely:

$\varepsilon=\frac{h}{T}$

Bai and Perron $(1998,2003)$ extend this approach to $F$ tests for 0 vs. $\ell$ breaks and $\ell$ vs. $\ell+1$ breaks with arbitrary but fixed $\ell$. Given an $m$-partition $i_{1}, \ldots, i_{m}$ the least squares estimates for the $\beta_{j}$ can easily be obtained. The resulting minimal residual sum of squares is given by:

$\operatorname{RSS}\left(i_{1}, \ldots, i_{m}\right)=\sum_{j=1}^{m+1} \operatorname{rss}\left(i_{j-1}+1, i_{j}\right)$

where $\operatorname{rss}\left(i_{j-1}+1, i_{j}\right)$ is the usual minimal residual sum of squares in the $j$ th segment. The problem of dating structural changes is to find the breakpoints $\hat{l}_{1}, \ldots, \hat{\imath}_{m}$ that minimize the objective function:

${ }^{1}$ By convention, $i_{0}=0$ and $i_{m+1}=n$. 


$$
R S S\left(\hat{\imath}_{1}, \ldots, \hat{\imath}_{m}\right)=\operatorname{argmin}_{\left(\hat{\imath}_{1}, \ldots, \hat{\imath}_{m}\right)} R S S\left(\hat{\imath}_{1}, \ldots, \hat{\imath}_{m}\right)
$$

Obtaining global minimisers by a grid search is computationally burdensome. Hierarchical algorithms have been proposed to do recursive partitioning or joining of subsamples (but these will not necessarily find the global minimisers). Bai and Perron (2003) present a version of that dynamic programming algorithm for pure and partial structural change models in an OLS regression context, which is adopted in strucchange. The basic idea is that of Bellman's principle: the optimal segmentation satisfies the recursion:

$R S S I_{m, n}=\min _{\left(m n_{h} \leq i \leq n-n_{h}\right)}\left[R S S\left(\mathcal{J}_{m-1, i}\right)+\operatorname{rss}(i+1, n)\right]$

Therefore, it suffices to know for each point $i$ the 'optimal previous partner' if $i$ was the last breakpoint in an $m$-partition. This methodology is valid under fairly general assumptions on regressors and disturbances.

\section{Empirical results}

Following the empirical approach outlined in the previous section, we analyse our data based on a set of salt based commodities presented in Table 1 using the following approaches (i) Zivot and Andrews (1992) (ii) Lee and Strazicich (2003, 2004 and (iii) Bai and Perron's (1998, 2003) multiple structural breakpoint test. Starting with our application of Zivot and Andrews (1992) these results are presented in Table 3.

Table 3: Zivot and Andrews Test Results (allowing one structural break)

\begin{tabular}{llll}
\hline Food product - description & Code & Test result & Break \\
\hline Low salt crisps & crisp.lowsalt (ts1) & $\mathrm{I}(0)$ & Week 62 \\
Standard crisps & crisp.standard (ts2) & $\mathrm{I}(0)$ & Week 51 \\
Low salt Kettle chips & kettlechips.lowsalt (ts3) & $\mathrm{I}(1)$ & - -- \\
Standard Kettle chips & kettlechips.standard (ts4) & $\mathrm{I}(0)$ & Week 50 \\
Low salt Tesco chips & tescochips.lowsalt (ts5) & $\mathrm{I}(1)$ & -- \\
Standard Tesco chips & tescochips.standard (ts6) & $\mathrm{I}(1)$ & -- \\
Unsmoked bacon & bacon.unsmoked (ts7) & $\mathrm{I}(1)$ & -- \\
Standard bacon & bacon.standard (ts8) & $\mathrm{I}(1)$ & -- \\
Unsmoked Butchers Choice bacon & butcherschoice.baconUS (ts9) & $\mathrm{I}(0)$ & Week 90 \\
Smoked Butchers Choice bacon & butcherschoice.baconS (ts10) & $\mathrm{I}(0)$ & Week 103 \\
Unsmoked Cookstown bacon & cookstown.baconUS (ts11) & $\mathrm{I}(1)$ & -- \\
Smoked Cookstown bacon & cookstown.baconS (ts12) & $\mathrm{I}(0)$ & Week 36 \\
Unsmoked Tesco bacon & tesco.baconUS (ts13) & $\mathrm{I}(1)$ & -- \\
Smoked Tesco bacon & tesco.baconS (ts14) & $\mathrm{I}(1)$ & -- \\
\hline
\end{tabular}

Note: The suffix 'S' denotes standard and 'US' denotes unsmoked

These results that allow for a single structural break suggest that there are six series which are stationary (low salt crisps, standard crisps, standard kettle chips, Butcher's Choice bacon unsmoked, Butcher's Choice bacon smoked and Cookstown bacon smoked, respectively) while the rest exhibit unit roots (they are non-stationary).

The breaks identified by the Zivot and Andrews procedure are the following, low salt crisps (week 62), standard crisps (week 51), standard kettle chips (week 50), Butcher's Choice bacon unsmoked (week 90), Butcher's Choice bacon standard (week 103) and Cookstown bacon standard (week 36). For all series excluding Butcher's Choice bacon unsmoked and Butcher's Choice bacon standard, the 
breaks occur after the second announcement round R2, while for unsmoked Butchers Choice bacon and smoked Butchers Choice bacon, the respective breaks are identified after both R2 and R3 (as shown in Table 2). A crucial factor that influences the interpretation of these breaks is the duration of these announcement effects. In other words, it is important to consider how long such announcements effects influence behaviour of consumers and at what stage do they damp down to an insignificant effect.

Next we consider results for the Lee and Strazicich's (2003) two break LM test on our data. These results are presented in Table 4.

Table 4: Lee and Strazicich LM unit root test with two breaks

\begin{tabular}{llll}
\hline Series & Break Dates & Lags (k) & t-stat \\
\hline Low salt crisps & 59,67 & 1 & $-7.13 *$ \\
Standard crisps & 26,68 & 0 & $-8.45 *$ \\
Low salt kettle chips & 61,67 & 1 & $-7.59 *$ \\
Standard kettle chips & 13,18 & 3 & $-6.80 *$ \\
Low salt Tesco chips & 48,79 & 1 & $-6.78 *$ \\
Standard Tesco chips & 48,83 & 0 & $-7.09 *$ \\
Unsmoked bacon & 43,66 & 0 & $-6.50 *$ \\
Standard bacon & 43,66 & 0 & $-6.52 *$ \\
Unmoked Butchers Choice bacon & 63,66 & 0 & $-6.50 *$ \\
Smoked Butchers Choice bacon & 63,66 & 0 & $-6.51 *$ \\
Unsmoked Cookstown bacon & 36,93 & 6 & $-5.82 *$ \\
Smoked Cookstown bacon & 25,44 & 0 & $-10.77 *$ \\
Unsmoked Tesco bacon & 43,66 & 0 & $-5.84 *$ \\
Smoked Tesco bacon & 36,66 & 0 & $-6.06 *$ \\
\hline
\end{tabular}

Note: Superscript $*$ denotes rejection of the null at the $5 \%$-level. Break dates refer to the relevant week in our sample.

Table 4 indicates results for the LM test allowing for two a priori unknown breaks. It also reports $k$ which is the number of lags selected and included in order to eliminate residuals serial correlation. Our results show that the unit root null hypothesis is always rejected at the 5\%-level for all fourteen series in our data set. However, despite the identification of breaks in the data an obvious limitation of this LM test procedure is that we assume that the number of breaks is known a priori and that the total number of breaks is strictly less than 3 . This limitation can be addressed by using the Bai and Perron procedure, for which results are presented in Table 5. We also present our Bai and Perron (1998, 2003) results graphically. ${ }^{2}$ Figures 1-5 indicate our results based on an application of such techniques for our fourteen salt based time series.

\section{\{Approximate Position of Figures 1 to 5\}}

Each figure indicates the location of relevant break-dates (in dotted lines), while the green coloured lines on the left panels indicate the first announcement round (R2, 10/10/2005 30/11/2005). The blue coloured lines on the left panels indicate the second announcement round (R3, 12/03/2007 - 14/05/2007). Thus, by direct visual examination, we can infer whether breaks occur before or after announcements are made. Each right hand panel indicates the optimal number of breaks identified by minimising RSS (as explained earlier) and the optimal number of

\footnotetext{
${ }^{2}$ Results have been obtained using $\mathrm{R}$ and the package strucchange.
} 
breaks are shown by the blue coloured lines. So each graphic indicates the optimal number of breaks and the location of each identified break point. ${ }^{2}$

Figure 1, top panel shows the results for low salt crisps (crisp.lowsalt, TS1). In the left panel, we can see in the period of the second announcement round (R2) demarcated clearly by the two vertical green lines. Similarly, the two vertical blue lines demarcate the time period of announcement round R3. The three dotted lines indicate the location of the three identified breaks. The right side of the top panel clearly shows that 3 optima breaks are identified based on minimising RSS. If we look at Figure 4, the middle panel for smoked Butcher's Choice bacon (butcherschoice.baconS, TS10), we can see announcement rounds R2 and R3 indicated by the blue and green lines respectively, while the vertical dotted lines indicate the three identified breaks. The right panel shows us that RSS is minimised when three breakpoints are identified, indicating that three breakpoints are optimal in this case. Likewise for the remaining twelve cases. $^{3}$

Table 5: Bai and Perron's $(1998,2003)$ multiple structural breakpoint test results

\begin{tabular}{lll}
\hline Food product - description & $\begin{array}{l}\text { Optimal number } \\
\text { of breaks }\end{array}$ & Description \\
\hline Low salt crisps & 3 & After R2, before R3 \\
Standard crisps & 4 & Before R2, 3 breaks between R2 and R3 \\
Low salt kettle chips & NA & N/A \\
Standard kettle chips & 4 & 3 breaks between R2 and R3 \\
Low salt Tesco chips & NA & N/A \\
Standard Tesco chips & NA & N/A \\
Unsmoked bacon & NA & N/A \\
Standard bacon & NA & N/A \\
Unsmoked Butchers Choice & 4 & After R2, before R3 \\
bacon & 4 & After R2, before R3 \\
Smoked Butchers Choice bacon & NA & N/A \\
Unsmoked Cookstown bacon & After R2, before R3 \\
Smoked Cookstown bacon & 3 & N/A \\
Unsmoked Tesco bacon & NA & N/A \\
Smoked Tesco bacon & NA & \\
\hline
\end{tabular}

As noted, the literature on consumer behaviour and advertising (i.e. Mela et al., 1997) strongly suggests that a period over 10-12 weeks is plausible for influencing consumption decisions, because in non-involved or low-involvement purchasing decisions there may be a degree of persistence in behaviour and consumption patterns may take a while to alter. If we assume a lag of up to 12 weeks as being relevant for altering consumption behaviour, it is clear that in the case of univariate time series allowing for a single break, the only relevant cases is Butcher's Choice bacon unsmoked (week 90), which is 'quite close' to, and after, the announcement period R3. So we have evidence pointing towards the significance of the breaks for Butcher's Choice bacon unsmoked. This suggests that health related announcement effects are potentially likely to have been significant for these two cases.

Overall our results suggest that, we can conclude that there is some limited evidence for the effectiveness of such announcements for the following commodities and breaks: standard crisps (week

\footnotetext{
${ }^{3}$ More detailed results for individual series such as TS1 (crisp.lowsalt) and TS10 (butcher- schoice.baconS) and all complete results are available from the authors
} 
18), standard kettlechips (week 18), Butcher's Choice bacon unsmoked (week 21), Butcher's Choice bacon standard (week 21). However, the most interesting results relate to Butcher's Choice bacon unsmoked and Butcher's Choice bacon smoked for week 79. Our data indicates significant structural breaks in week 79 for both Butcher's Choice bacon unsmoked and Butcher's Choice bacon smoked. This ties in well with our evidence based on the Zivot and Andrew's test and implies that for Butcher's Choice bacon unsmoked there is strong evidence linked a significant break which follows the two announcements in periods R2 and R3. For series Butcher's Choice bacon smoked, the same conclusion can be reached on the basis of the Bai and Perron procedure.

\section{Discussion and Conclusions}

This paper has employed leading UK supermarket loyalty card data which records information on purchase decisions by consumers in order to assess the effectiveness and impact of the UK reduced salt campaign. We present an empirical analysis of consumption data to assess the effectiveness of the UK FSA 'reduced salt campaign' with a specific focus on health related announcements undertaken by the FSA during its 'low salt campaign'. Econometrically, we adopted a general approach to determining structural breaks in consumption data, including making use of minimum LM unit root tests whereby structural breaks are endogenously determined from the data.

Our analysis reveals some limited evidence supporting the effectiveness of the FSA's reduced salt campaign. Overall, our analysis points towards empirical evidence for structural breaks in consumption data for six commodities following health related announcements and these results are reasonably strong for two commodities, Butcher's Choice bacon unsmoked and Butcher's Choice bacon standard. For these two products we can see evidence of structural breaks that correlate with two of the announcement periods of the policy.

Although these results are only for a limited sub-set of the products examined they do support the findings of with Lui et al. (2014) who report that information based policies typically have a modest influence consumer choice and behavior. They also indicate that there is a need for a dynamic examination of the impact of information provision programmes if the potential lag effects are to be identified. In addition, our results suggest that for most of the salt rich foods we consider there is no statistically identifiable change in trends in consumption. These results therefore support the findings of Griffith et al. (2017) who argue that reductions in salt that have been identified in health survey data are most likely as a result of product reformulation and not necessarily product substitution by consumers.

A potential course of future research in this area would be to examine if there are differences in trends in consumption for products that have not been reformulated versus those that have. This analysis would help us further understand the channel through which reductions in salt consumption are occurring. The importance of this type of analysis with a focus on prepared foods is required as the proportion of prepared food consumed by households increase. Similarly, it would be interesting to conduct related analysis on food consumed away from the home which is also growing as proportion of overall food consumption.

In terms of limitations of the research presented there are two obvious issues. First, the time period considered is not as long as we would like. Therefore, any future analysis of this type would be improved if longer time series of data could be employed. Secondly, the scope of products examined is somewhat limited and extending the product coverage would be beneficially. Finally, another limitation with this analysis that will not occur with more recent data is that the period we considered was not subject to a uniform TLS which would make the translation of the information about salt consumption at the point of purchase more complicated. In addition, Tesco during the period we examine adopted a variant of the TLS with a stronger emphasis on the Guideline Daily Amount (GDA) by nutrient which might have had a downward bias on the impact of the information campaign. With a longer, more widely sourced set of products drawn from more recent time periods these types of limitations could be overcome in future research. 


\section{References}

Andrews, D W K. Tests for parameter instability and structural change with unknown change point. Econometrica, 61(4):821-856, Jun 1993.

Bai, J. and Perron, P. Estimating and testing linear models with multiple structural changes. Econometrica, 66(1):47-78, Jun 1998.

Bai, J. and Perron, P. Computation and analysis of multiple structural change models. Journal of Applied Econometrics, 18:1-22, Feb 2003.

Balcombe, K., I.M. Fraser and S. Di Falco (2010). Traffic Lights and Food Choice: A Choice Experiment Examining the Relationship Between Food Labels and Price, Food Policy. 35(3): 211-220.

Basher, S. and Mohsin, M. PPP tests in cointegrated panels: evidence from asian developing countries. Applied Economics Letters, 11(3):163-166, Jan 2004.

Collins, M., Mason, H., O’Flaherty, M., Guzman-Castillo, M., Critchley, J. and Capewell, S. An Economic Evaluation of Salt Reduction Policies to Reduce Coronary Heart Disease in England: A Policy Modeling Study, Value in Health, 17(5): 517-524, 2014.

Fulponi, L. Private voluntary standards in the food system: The perspective of major food retailers in OECD countries. Food Policy, 31(1):1-13, 2006.

Golan, E. and Unnevehr, L. Food product composition, consumer health, and public policy: Introduction and overview of special section. Food Policy, 33(6):465-469, 2008.

Griffith, R., O'Connell, M. and Smith, K. The Importance of Product Reformulation Versus Consumer Choice in Improving Diet Quality. Economica, 84: 34-53, 2017.

He F.J. and MacGregor, G.A. A comprehensive review on salt and health and current experience of worldwide salt reduction programmes. Journal of Human Hypertension, 23: 363-84, 2009.

Lee, J. and Strazicich, M.C. Minimum lagrange multiplier unit root test with two structural breaks. Review of Economics and Statistics, 85(4):10821089, Dec 2003.

Lee, J., List, J.A. and Strazicich, M.C. Non-renewable resource prices: Deterministic or stochastic trends? Journal of Environmental Economics and Management, 51:354-370, Apr 2006.

Liu, P. J., Wisdom, J., Roberto, C. A., Liu, L. J. and Ubel, P. A. Using behavioral economics to design more effective food policies to address obesity. Applied Economic Perspectives and Policy, 36(1), 6-24, 2014.

Mela, C.F., Gupta, S., and Lehmann, D. R. The long term impact of promotion and advertising on consumer brand choice. Journal of Marketing Research, 34, 1997. 
Nghiem, N., Blakely, T., Cobiac, L.J., Pearson, A.L. and Wilson, N. Health and Economic Impacts of Eight Different Dietary Salt Reduction Interventions. PLoS ONE, 10(4): e0123915. doi:10.1371/journal.pone.0123915, 2015.

Shankar, B., Brambila-Macias, J., Traill, B., Mazzocchi, M. and Capacci, S. An Evaluation Of The UK Food Standards Agency's Salt Campaign. Health Economics, 22: 243-250, 2013.

$\mathrm{Ng}, \mathrm{S}$. and Perron, P. Unit root tests in arma models with data-dependent methods for the selection of the truncation lag. Journal of the American Statistical Association, 90(429):268-281, 1995.

$\mathrm{Ng}, \mathrm{S}$ and Perron, P. Lag length selection and the construction of unit root tests with good size and power. Econometrica, 69(6):1519-1554, 2001.

Sutherland, J., Edwards, P. Shankar, B. and Dangour, A.D. Fewer adults add salt at the table after initiation of a national salt campaign in the UK: a repeated cross-sectional analysis. British Journal of Nutrition, 110:552-558, 2013.

Van Camp, D., de Souza Monteiro, D.M. and Hooker, N.H. Stop or go? How is the UK food industry responding to front-of-pack nutrition labels? European Review of Agricultural Economics, 39(5):821-842, 2012.

Webb, M., Fahimi, S., Singh, G.M., Khatibzadeh, S., Micha, R., Powles, J. and Mozaffarian, D. Cost effectiveness of a government supported policy strategy to decrease sodium intake: global analysis across 183 nations. The British Medical Journal, 356:i6699, 2017.

World Health Organization. Global Status Report on Non-Communicable Diseases, 2014.

http://apps.who.int/iris/bitstream/handle/10665/148114/9789241564854_eng.

Zivot, E. and D. Andrews, D. Further evidence of great crash, the oil price shock and unit root hypothesis, Journal of Business and Economic Statistics, 10, 251-270, 1992.

Figure 1: Bai and Perron Test Results (L) and Optimal Breakpoints (R): 
RE test (recursive estimates test)

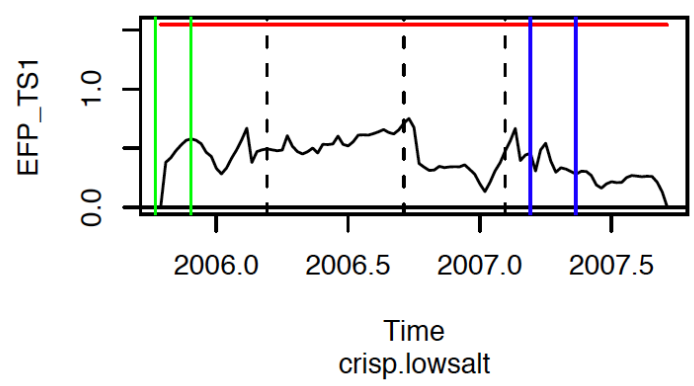

RE test (recursive estimates test)

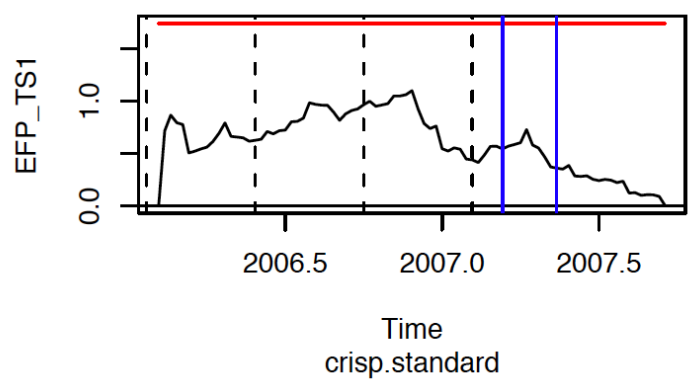

RE test (recursive estimates test)

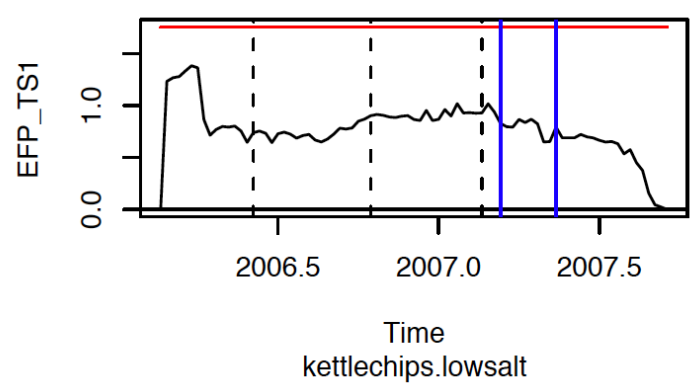

BIC and Residual Sum of Squares

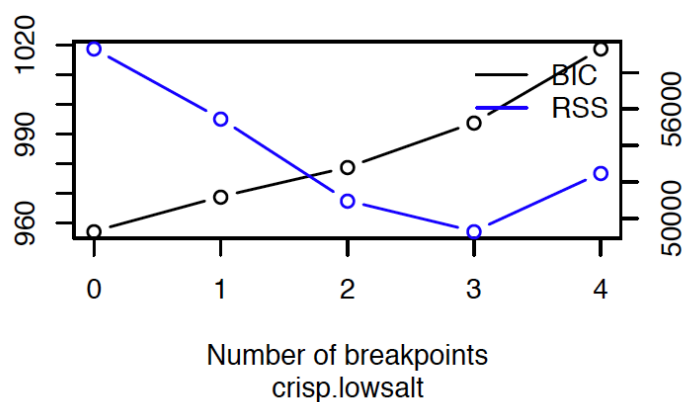

BIC and Residual Sum of Squares

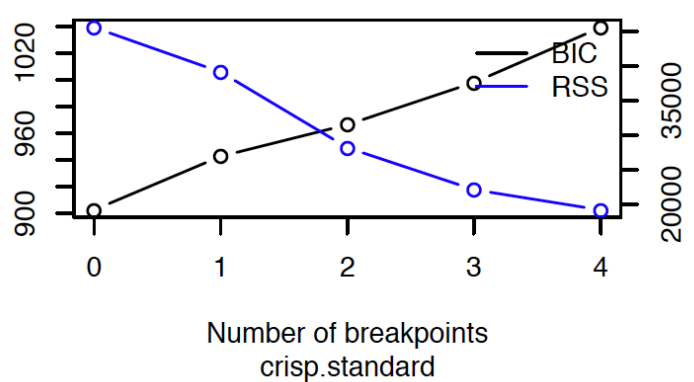

BIC and Residual Sum of Squares

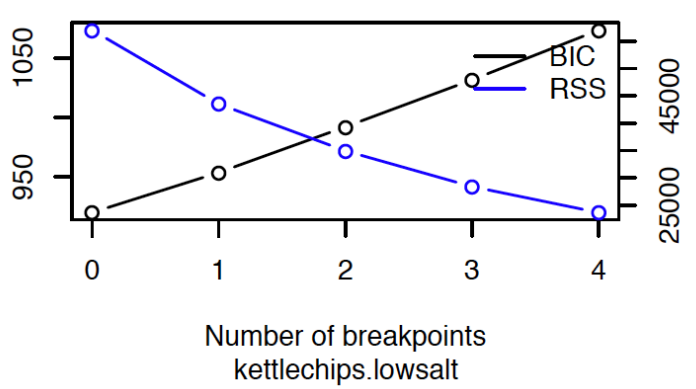

Figure 2: Bai and Perron Test Results (L) and Optimal Breakpoints (R): 
RE test (recursive estimates test)

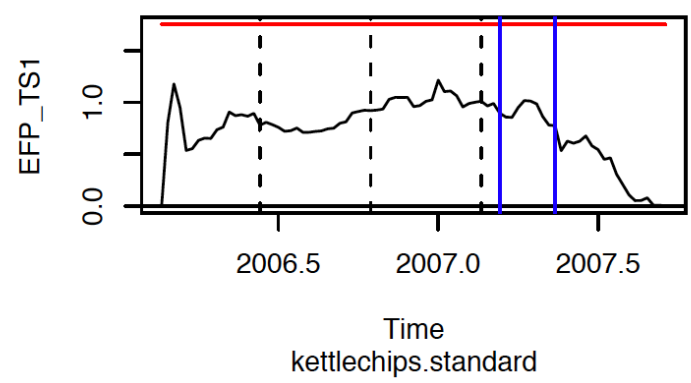

RE test (recursive estimates test)

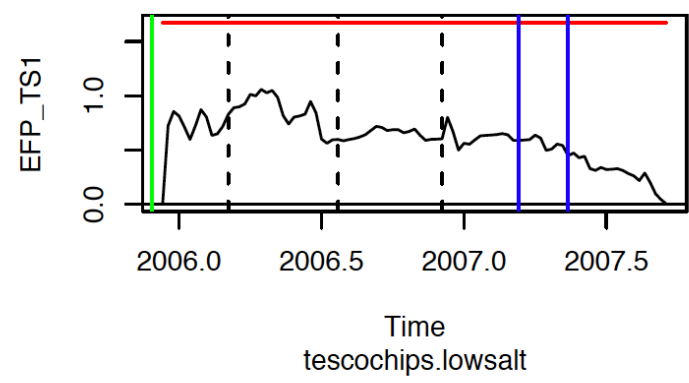

RE test (recursive estimates test)

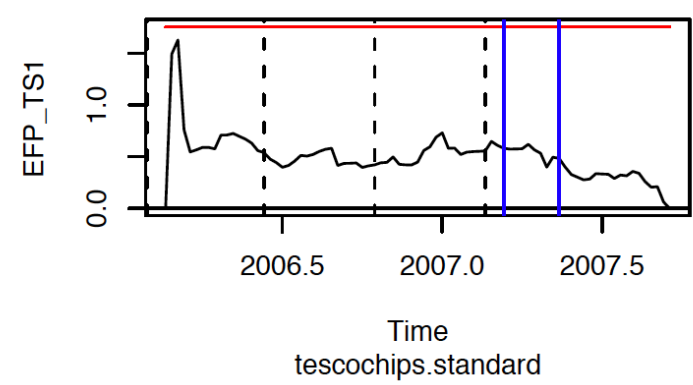

BIC and Residual Sum of Squares

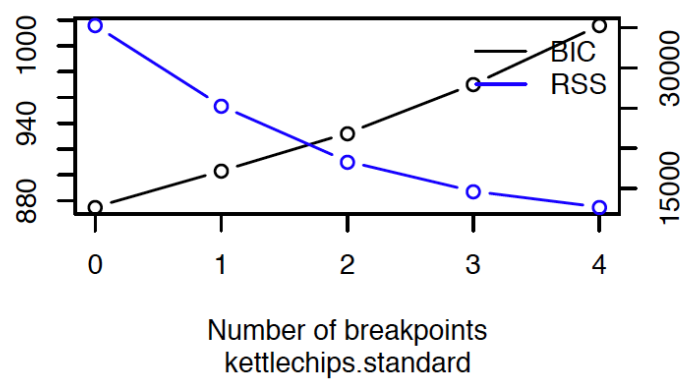

BIC and Residual Sum of Squares

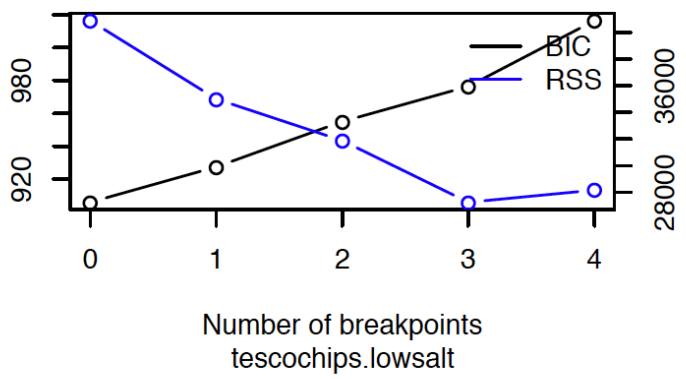

BIC and Residual Sum of Squares

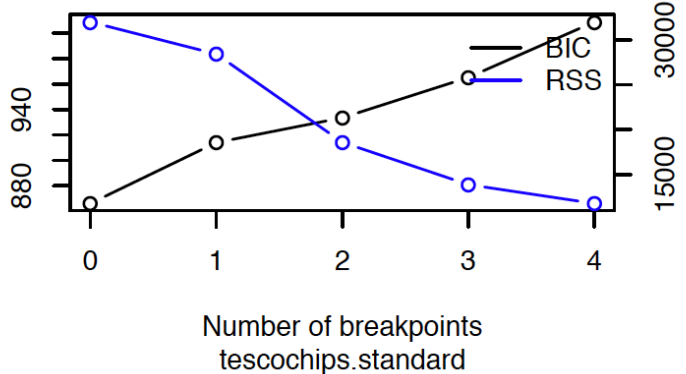

Figure 3: Bai and Perron Test Results (L) and Optimal Breakpoints (R): 
RE test (recursive estimates test)

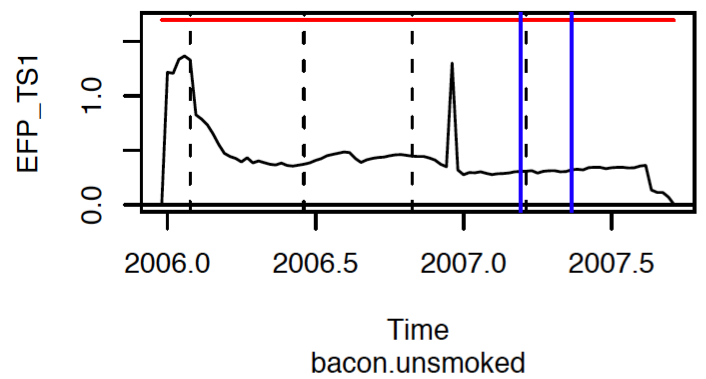

RE test (recursive estimates test)

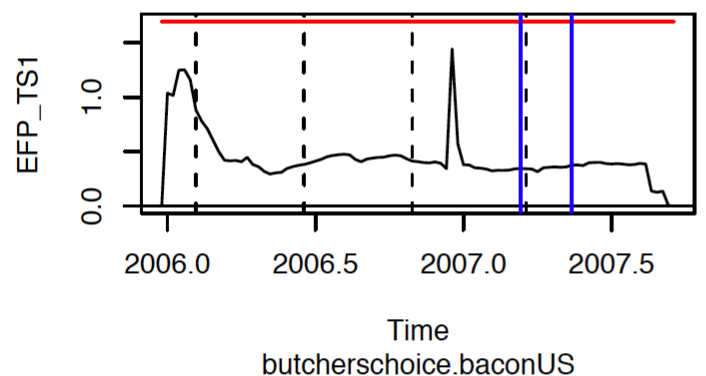

RE test (recursive estimates test)

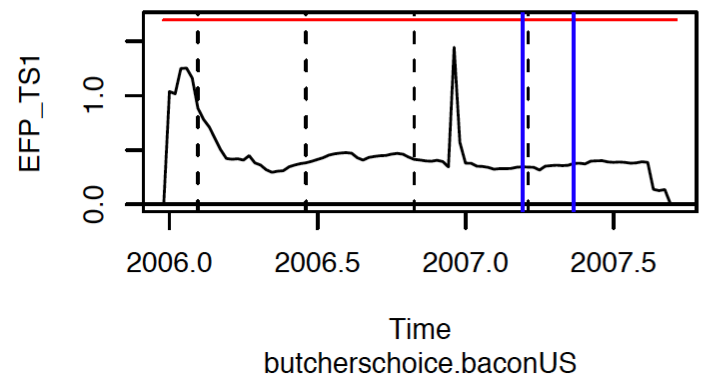

BIC and Residual Sum of Squares

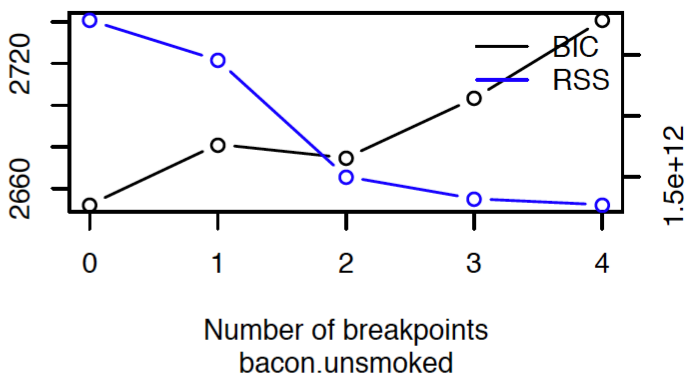

BIC and Residual Sum of Squares

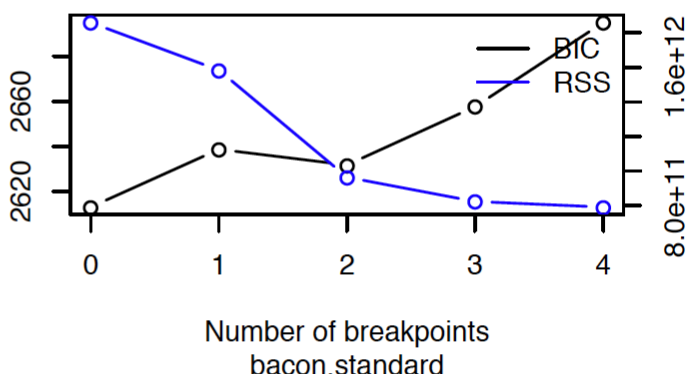

BIC and Residual Sum of Squares

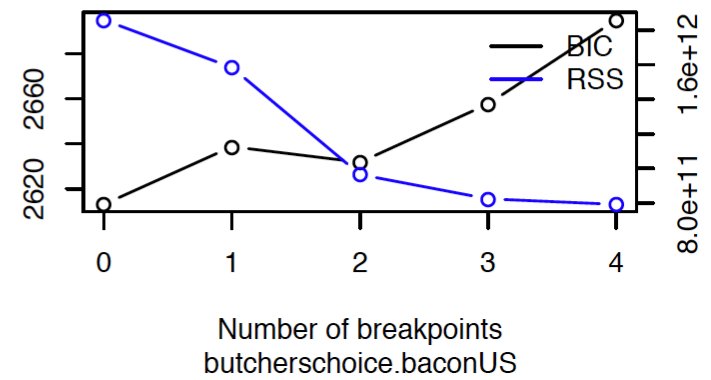

Figure 4: Bai and Perron Test Results (L) and Optimal Breakpoints (R): 
TS10-TS12 (top to bottom)

RE test (recursive estimates test)

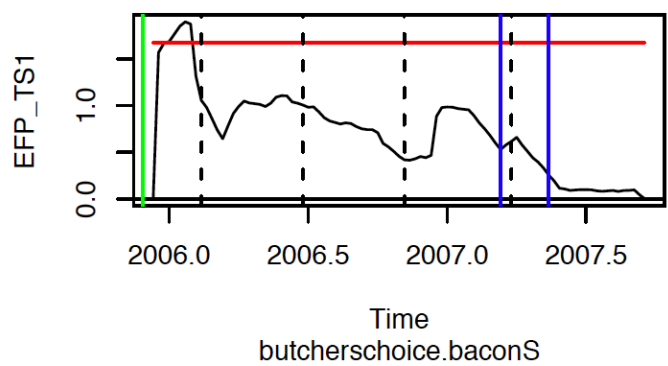

RE test (recursive estimates test)

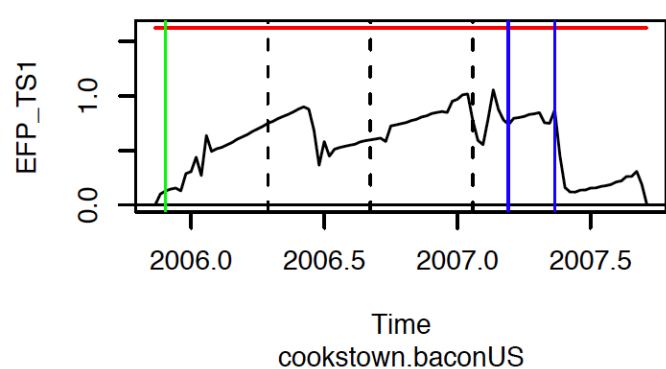

RE test (recursive estimates test)

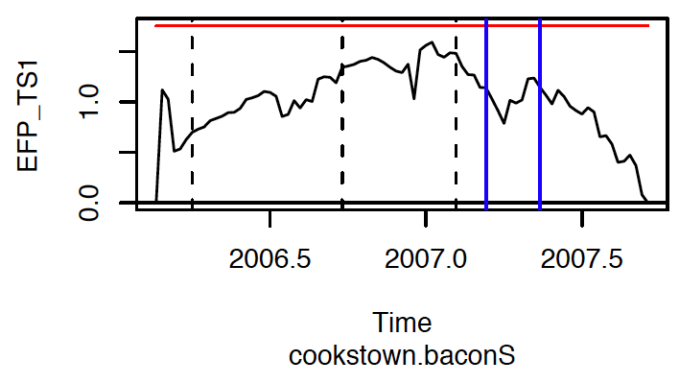

BIC and Residual Sum of Squares

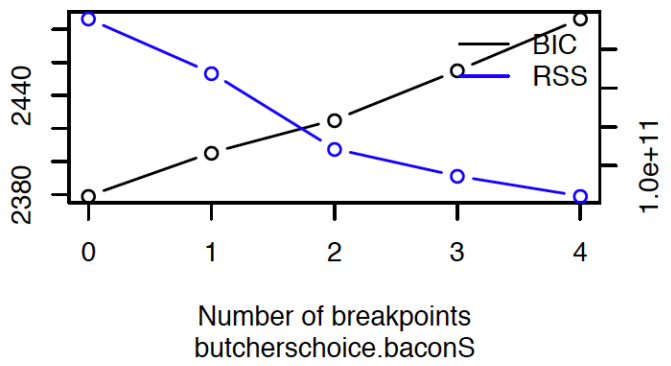

BIC and Residual Sum of Squares

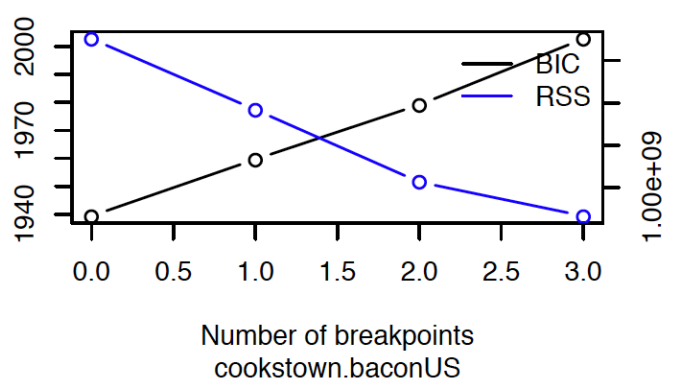

BIC and Residual Sum of Squares

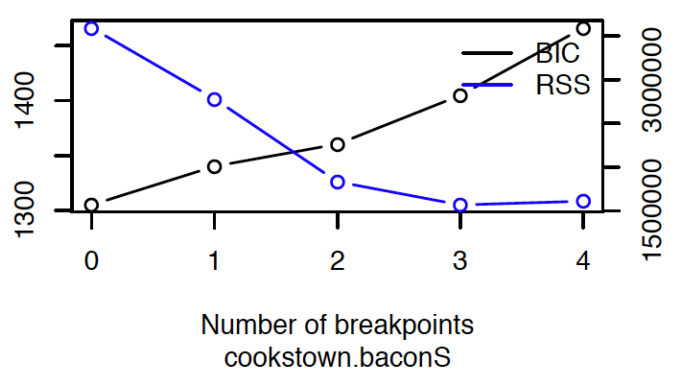

Figure 5: Bai and Perron Test Results (L) and Optimal Breakpoints (R): 
TS13-TS14 (top to bottom)

RE test (recursive estimates test)

BIC and Residual Sum of Squares

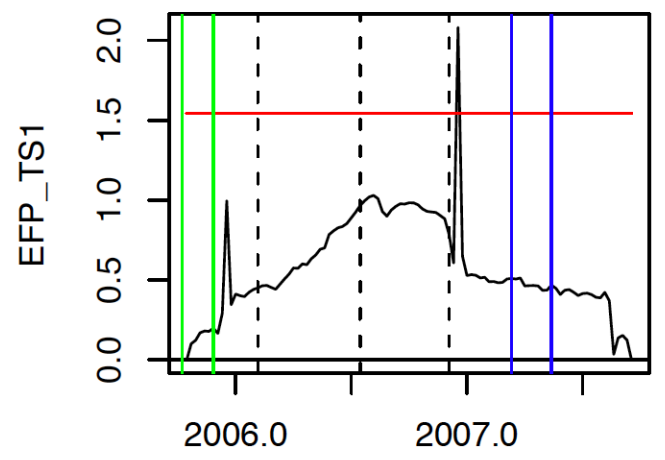

Time

tesco.baconUS

RE test (recursive estimates test)

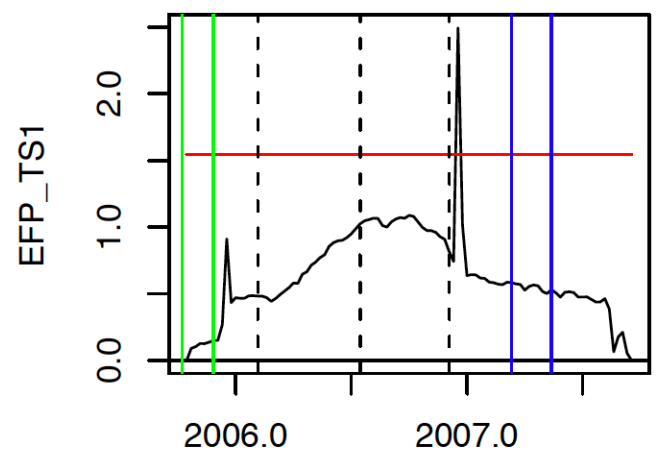

Time

tesco.bacons

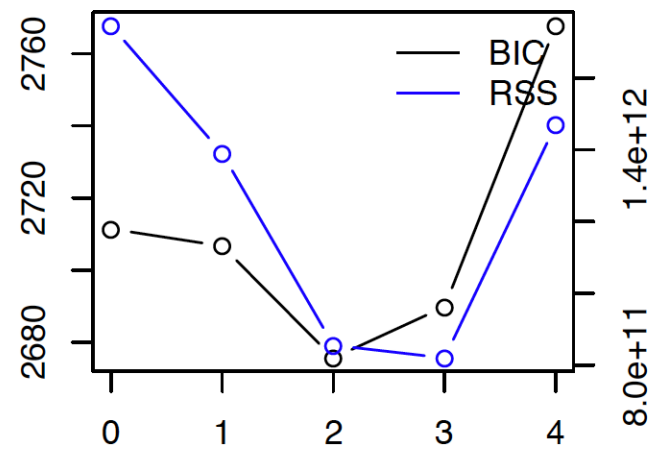

Number of breakpoints tesco.baconUS

BIC and Residual Sum of Squares

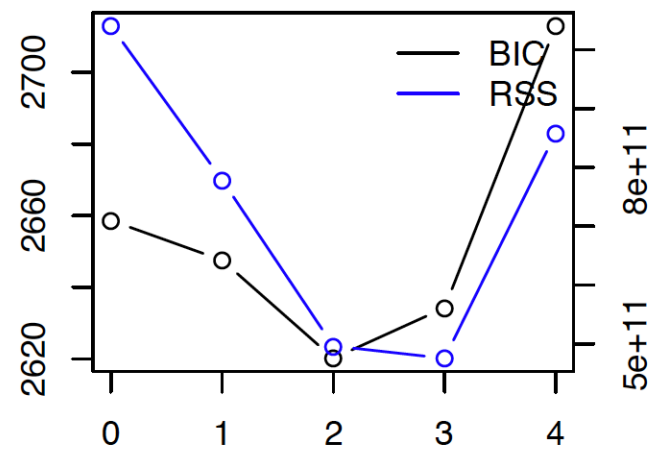

Number of breakpoints tesco.bacons 